\title{
Election Results Prediction System based on Fuzzy Logic
}

\author{
Harmanjit Singh \\ Research Scholar, \\ Singhania University, \\ Rajasthan, INDIA
}

\author{
Gurdev Singh, Ph.D \\ Professor, Deptt of Information \\ \& Technology \\ Gurukul Vidyapeeth Institute of \\ Engineering \& Technology \\ Banur, Chandigarh
}

\author{
Nitin Bhatia \\ DAV College, Jalandhar \\ Punjab, INDIA
}

\begin{abstract}
Election is very popular word in the universe. As per the definition, Election is a process to select suitable from a group of candidates. But the process and properties may vary for different sectors. It has different types. Like local election, legislative election, parliamentary election, presidential election, senate election or election in a small group / union. As election is very famous same way election prediction is again not a new keyword. It has same long life as election. But still there is a challengeable task to predict accurate result. The use of fuzzy logic in social science to evaluate the prediction is the core part of this paper. A toolbox from MATLAB software named fuzzy logic toolbox is used for this purpose. Some input arguments are being considered which scaled using linguistic variables to predict the chances of winning along with chances of losing of a candidate.
\end{abstract}

\section{Keywords}

Fuzzy Logic, Mamdani, Election Prediction Evaluator, Membership Function, Linguistic Variable

\section{INTRODUCTION}

It is the fact that area of political science is strongly based upon vague and uncertain statements. The concept of election prediction which comes under political science is also affected from the non reasonable statement. Generally it assumes that if a candidate having high popularity will have a very strong chance to win. But what will happen, if all the candidates have very high popularity in same constituency or all have very low popularity. Will they all win the election? So to predict the election based upon one or two factors or non favorable factors is not up to mark. Fuzzy logic makes it easy and possible to predict the election in a realistic way. It also allows the qualitative and subjection analysis.

This work is based upon uncommon use of intelligence because it is related to that area which is not generally used by researches. The concept of fuzzy logic is very popular in traditional sectors like engineering, medicine, stock market, aircraft control, weather forecasting and other decision making areas of computer science branches as well as non computer science branches. Politics is a vital part of social science. This can also become the famous area for research. If we will calculate accurate results via some software's / tools. Social science deals with complexity. Lots of variables are used which are not so easy to simplify and to obtain accurate result. It generally deals via traditional way. i.e Public Opinion. In simple term, majority of the values of these variables are derived from only public opinion. So in this research, it has experimented and explained the importance and use of fuzzy logic in social science. So, fuzzy logic is a suitable approach to understand this kind of imprecision. This is the first reason to use Fuzzy Logic in this research.

Second reason to use fuzzy logic because fuzzy logic also helps to generate a model based approach which is used in politics. At the end of this paper, a model is presented along with the answer (prediction) closer to the actual result. It is quite easy to explain and understand the process of reasoning and decision making.

The main motive of this work is to only present the feasibility and reliability of a system that will help in political decision accurately. The final result corresponds to the nearby prediction of a candidate of any level of election like local, state or center. It others words this model can be used in election for Councilor, Members of legislative assembly, Members of parliaments, Presidential election and Senate election in universities etc. This system is basically defined by an opinion sampling and through successive discussions with the experts along with people who are in politics.

The lateral sections of this works are designed as follows 2Related Work, 3-Proposed Work, 3.1-Design Methodology, 3.2-Input Membership Functions, 3.3-Output Membership Function, 4-Result Analysis, 5-Conclusion and Future Work and at last 7- References

\section{RELATED WORK}

Fuzzy logic is capable to handle problems in vague statement, to imprecise data and also in decision making system (DMS). Professor L. A. Zadeh has invented the concept of Fuzzy Logic in 1964. He presented his first paper on fuzzy set in 1965 [1]. Later on, Assilian and E. Mamdani developed first fuzzy logic controller in 1974 in United Kingdom. Due to its reliability, capability researchers are using this concept for designing, developing and implementing algorithms as well as the working models with the help of fuzzy controller. Along with fuzzy logic, Genetic Algorithm (GA) is also used to design and implement with fuzzy logic controllers to make models and application [2]. Fuzzy also helps to solve different real world due to its systematic rule depended concept. J. M. Mendal says that fuzzy logic system as a non linear mapping of an input data vector into a scalar output [3]. Theory of rough set discussed about the different kind of formulation and interpretations. This is also the part of fuzzy [4]. The similarities and differences between rough set theories and fuzzy sets with respect to two formulation of fuzzy set and two views of rough sets are explained by Yao in 1998 [5]. Different kind of applications based upon fuzzy logic / fuzzy set explained by Davidson and Harward in 2003 [6]. Fuzzy rules plays vital role in fuzzy inference system (FIS) and the same approach from latest point of view are verify to provide 
the helps to learn such rule based model for any kind of difficult task is discussed by Wang and Brigde in 2000 [7].

Neural learning with Fuzzy Inference System (FIS) is again a wide concept which is also used for decision making. It shows the reliability of FIS. It gives the transformation from more than one independent variable to only one output using fuzzy logic toolbox in MATLAB. In FIS numbers of rules are there based on "if-then" conditions. These rules are very smooth to learn, smooth to use and can be converted according to the scenario. They help to find out the conclusion easily. A. Abraham has explained FIS also with neural network techniques in distinct ways in 2008 [8]. Along with Fuzzy, Neural and Neuro Fuzzy Inference System (NFIS) are there to empower the importance of FIS. This parallel study was discussed by M. Z. Shafiq et al. in 2008 for Portscan Detection [9]. How multi-decision making system can be formulated via fuzzy? The concept was fabricated in 2004 by Zhiyi's formula based on Mamdani type FIS to assess compost maturity and stability [10]. A fuzzy logic based application to predict risk in loan providing organizations was developed and tested on different financial organizations by Kapoor and N. Bhatia in 2011 [11]. How the software risks can be calculated by using fuzzy logic? The answer of this question was given by again Kapoor and N. Bhatia in 2011 [12]. Gursharan Singh and N. Bhatia developed a fuzzy logic based cricket player performance evaluator in 2011. 96 rules were designed in FIS to check the performance of a player via fuzzy logic. There was positive as well as negative performance was there in this research. They also used input and output membership functions in the research [13].

Smithson [14] describes the theory of FL along with Fuzzy Set in Psychology. His theory was not only in one particular sector but in several fields as perception and memory theories to solve measurement problems. There is another researcher J. Russell, he has contributed the logic for facial emotion recognizer based on fuzzy logic [15]. Now the major role to provide the importance of FL in social sector was given by Dimitrov in 1997. He has given the practicality of fuzzy logic to deal with paradoxical and chaotic nature of social system [16]. This kind of system provides the help to deal with opposite opinions. Yamashita's in 1998 provides the concept of fuzzy system in the context of decision aid. After the fuzzy reasoning applied on the input values supplied by the students the system acts as a kind of vocational guidance [17]. Lius Teran introduced new concept in social science which was totally web based Voting Assistance Application (VAA) used to aid voters for finding the party or candidate that is most in line with their choice in 2011. This is treated as the latest concept in the field of social science [18]. Campbell and coworkers [19-21], Lewis-Beck and coworkers [22-24] have presented their typical contribution in the sector of presidential election forecasting with the help from political scientists. Along with political scientists, an economist, Fair [25] also generated some models which were based on economic variables. This has given the path and scope for golden era for researches to implements the concepts of social science along with the fuzzy logic technique.

Royes and Bastos [26] in 1988 used the fuzzy concepts to build a fuzzy expert system based on the vagueness and imprecision of the statement. Those investigators concluded that the outstanding feature of political science is reasoning based on imprecise statements and vague concepts. They also provided an example for predicting the chance of re-election using fuzzy logic
Due to the efforts and the practical utility of these above applications in the field of social science along with fuzzy logic it clearly indicates that how much woks has done and still there is a scope of improvement to provide the better result in the term of prediction. This kind of work is provided in this paper with some result analysis along with the chances of winning and chances if losing, if any. Because prediction play an important role in election and it is the demand of time to provide the accurate prediction by using tools or technology.

\section{PROPOSED WORK}

In this research work, the concept of fuzzy-logic inference system is used for predicting the result of an election. Fuzzy Inference Systems (FIS) use fuzzy sets and "if-then" rules relevant to fuzzy sets to make decisions about incomplete or vague information. The same concept was used by Riley in 2005 to find out the behavior of robot soccer [27]. There are basically Mamdani and Sugeno, two most commonly fuzzy inference systems are present in MATLAB. In this research work, Mamdani algorithm is used to determine the output. FIS system executes in three major steps: Fuzzification, Inference and Defuzzification [28]. Defuzzification provides the information in term of values and the same can be used to predict the result. The whole research is divided into some number of phases. In first phase, the degree of membership for crisp input variables is determined. At second phase, i.e. inference level, evaluation of fuzzy rules is done and output is produced for each rule. At last, the resulting fuzzy output is converted back into physical values through a defuzzfication process. In the first stage twenty two parameters were discussed for input variables but after discussion with some experts and removing/grouping some dependent arguments only nine parameters are selected for this research work as input variable and one for output. 91 rules have designed and made for this fuzzy system. Each input has three membership functions except for currently on govt. and party/independent which has two membership functions. The output has five membership functions. The GUI tool of MATLAB is used to develop the proposed predictor.

\subsection{Design Methodology}

In this fuzzy system, nine parameters as linguistic variables are used that affect the winning chance of a candidate or a party. The arguments which are taken for this research are: No Of Years In Active Politics, Currently In Government, Performance In Development, Inclination Of Voters, Vote Bank, Internal War, Party / Independent, and Major Issue. Winning Chance is taken as an output parameter. All these input variables actually affect the entire election system and these are also the part of that system which is used for prediction purpose in case of public opinion. Figure 1 shows the input variables and output variables.

On the basis of the description of input and output variables 91 rules are defined. Following is the description of some of the rules:

Rule 1: If (NoOfYears is Low) and (CurrInGovt is Yes) and (DevPerf is Low) and (Popularity is Low) and (Inclination is Low) and (VoteBank is Against) and (IWar is Partially) and (Part_Ind is Party) and (MajorIssue is Against) then (WinningChance is VeryLow) (1).

Rule 2: If (NoOfYears is Medium) and (CurrInGovt is No) and (DevPerf is Low) and (Popularity is High) and 
(Inclination is Medium) and (VoteBank is Infavour) and (IWar is No) and (Part_Ind is Party) and (MajorIssue is NoSuchIssue) then (WinningChance is Low) (0.45)

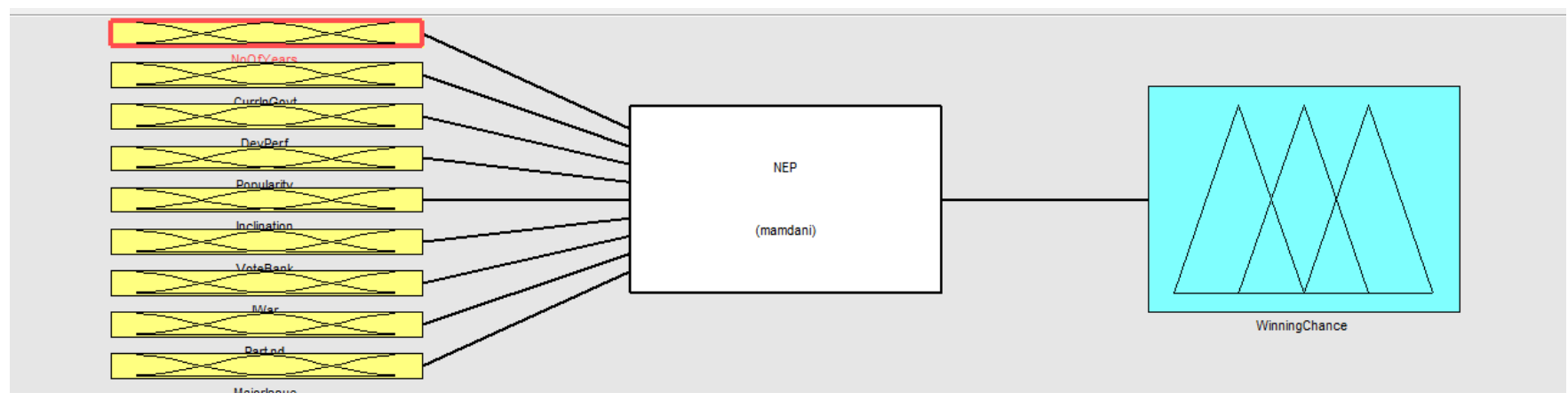

Fig. 1: Nine Inputs and One Output Parameter.

Rule 3: If (NoOfYears is Low) and (CurrInGovt is No) and (DevPerf is High) and (Popularity is Low) and (Inclination is High) and (VoteBank is Against) and (IWar is Partially) and (Part_Ind is Party) and (MajorIssue is NoSuchIssue) then (WinningChance is Medium) (0.6)

Rule 4: If (NoOfYears is Medium) and (CurrInGovt is Yes) and (DevPerf is Medium) and (Popularity is Low) and (Inclination is Medium) and (VoteBank is Against) and (IWar is Fully) and (Part_Ind is Independent) and (MajorIssue is InFavour) then (WinningChance is Low) (0.7)

Rule 89: If (NoOfYears is Low) and (CurrInGovt is No) and (DevPerf is Low) and (Popularity is Low) and (Inclination is Medium) and (VoteBank is Infavour) and (IWar is No) and (Part_Ind is Independent) and (MajorIssue is Against) then (WinningChance is Low) (0.45)
Rule 90: If (NoOfYears is Medium) and (CurrInGovt is No) and (DevPerf is Medium) and (Popularity is High) and (Inclination is High) and (VoteBank is Infavour) and (IWar is Fully) and (Part_Ind is Independent) and (MajorIssue is Against) then (WinningChance is VeryLow) (0.4)

Rule 91: If (NoOfYears is High) and (CurrInGovt is Yes) and (DevPerf is High) and (Popularity is High) and (Inclination is Low) and (VoteBank is Infavour) and (IWar is No) and (Part_Ind is Independent) and (MajorIssue is NoSuchIssue) then (WinningChance is High) (0.15)

\subsection{Input Membership Functions}

There are nine input variables (NoOfYears, CurrInGovt, DevPerf, Popularity, Inclination, VoteBank, IWar, Part_Ind and MajorIssue taken in this research based on fuzzy system. These variables use different membership functions.

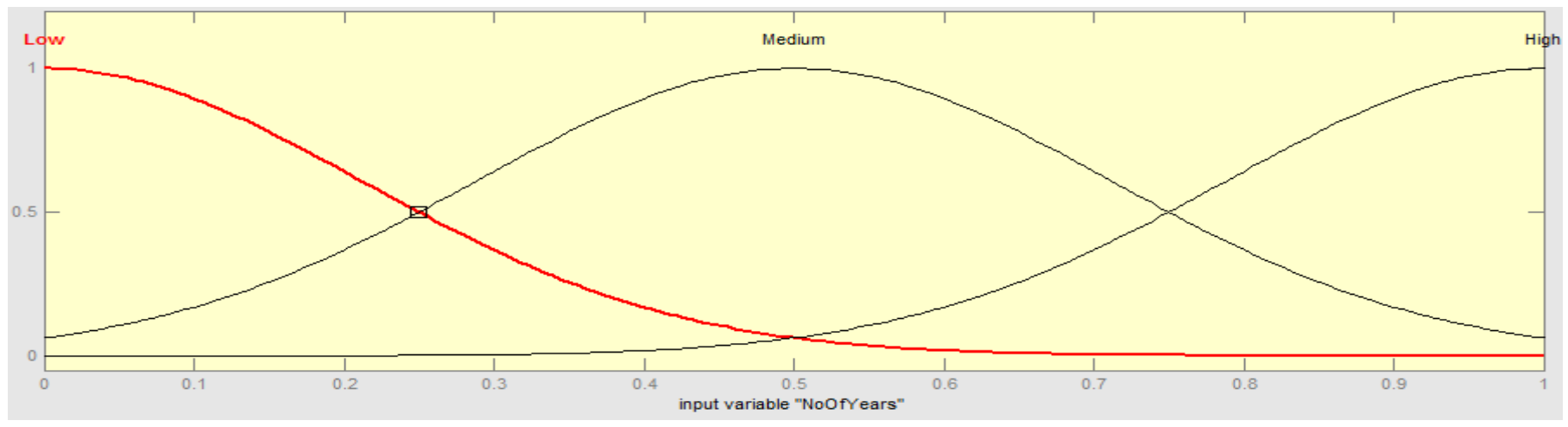

Fig. 2: Membership Functions for input Variable NoOfYears.

For instance, noofyears has three membership functions to represent Low, Med and High as shown in Figure 2. These functions are guassmf for Low, Medium and High. On the other hand CurrInGovt has two membership functions to represent the Yes or No. These functions are smf for Yes and zmf for $N o$. zmf: Z-shaped built-in membership function (zmf) is used to define the variable No. The weight is calculated by the following formula: 


$$
f(x ; 0,0.5)=\left\{\begin{array}{c}
1, x \leq 0 \\
1-2\left(\frac{x-0}{0.5-0}\right)^{2}, 0 \leq x \leq \frac{0+0.5}{2} \\
2\left(\frac{x-0.5}{0.5-0}\right)^{2}, \frac{0+0.5}{2} \leq x \leq 0.5 \\
0, x \geq 0.5
\end{array}\right\}
$$

gaussmf: Gaussian curve built-in membership function is used to define the variable Low, Medium and High. The weight is calculated by the following formula:

$$
f(x ; 0.2123,0.5)=e^{\frac{-(x-0.5)^{2}}{2(0.2123)^{2}}}
$$

smf: S-shaped built-in membership function is defined for variable Yes. The weight is calculated by the following formula:

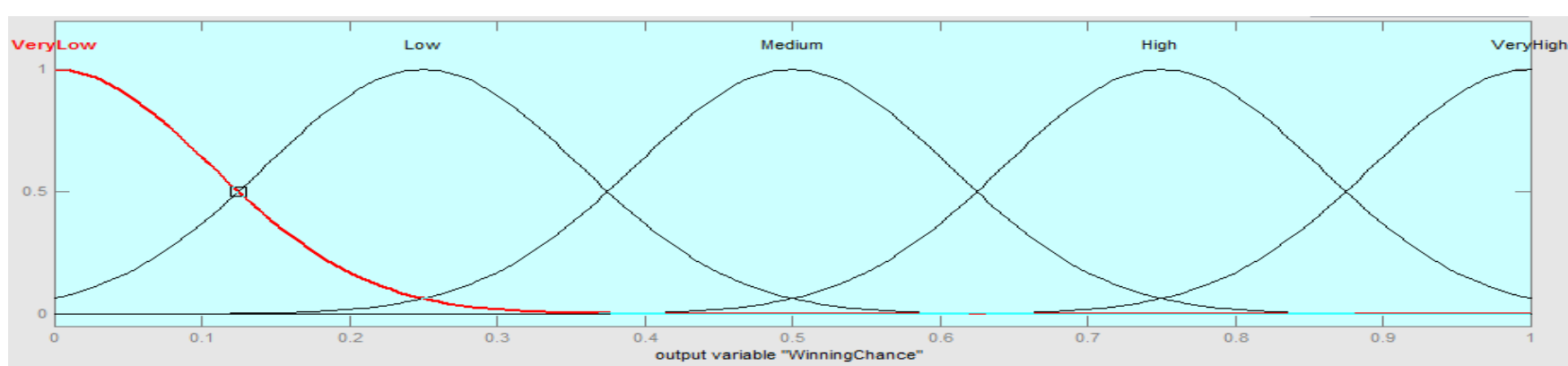

Fig. 3: Membership Functions for Output Variable WinningChance.

\section{RESULT ANALYSIS}

To calculate the prediction of a candidate by using the prediction evaluator, there are five different hypothetical scenarios as below.

Scenario 1: A candidate has low no of years in active politics and currently he belongs to ruling party but he has low performance in development and popularity. There is no impact of vote bank major issues are in favor. There is no internal war in the party. All these arguments are entered into the fuzzy logic based prediction system in MATLAB fuzzy logic toolbox [28], shown in Figure 4 (a). The output of this scenario shows that it has winning chances of $67 \%$ as shown in Figure 4 (b).

\subsection{Output Membership Function}

There is one variable named winningchance is used as an output variable which has five levels: Very Low, Low, Medium, High and Very High as shown in Figure 3. All these five levels are defined by the membership function gaussmf.

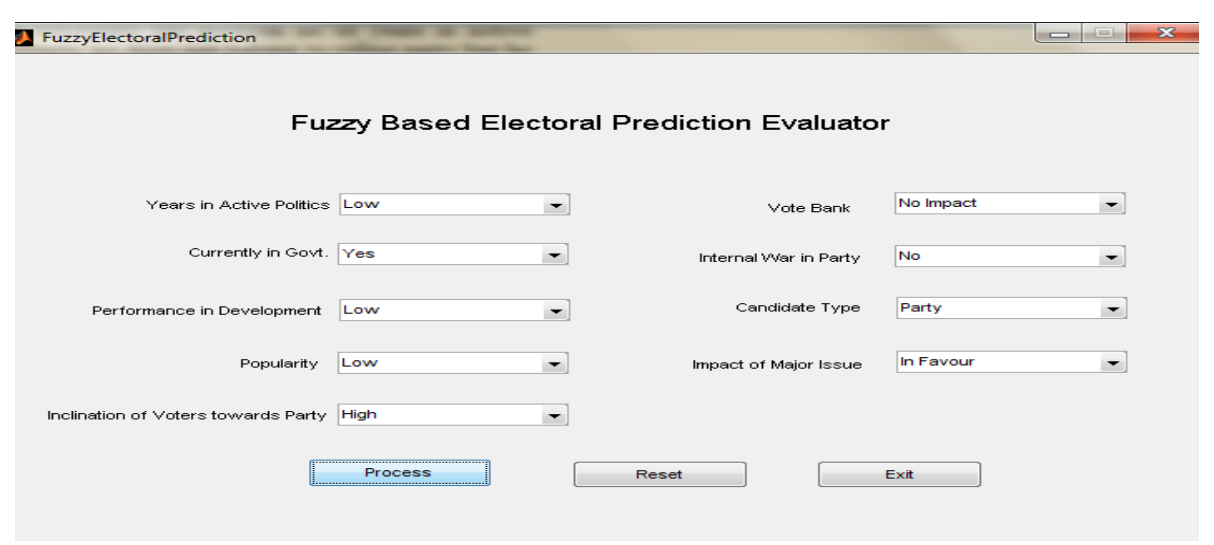

(a)
Scenario 2: A candidate has medium no of years in active politics and currently he does not belong to ruling party but he has high performance in development as compared to scenario 1 , popularity and vote bank is also in his favor and he acts as a candidate of party not independent candidate. There is a little internal war in the party and all the major issues are in his favor. Due to the high performance of development, popularity and high inclination of voters towards party the chances of winning are very high than previous case. All these arguments are entered into the fuzzy logic based prediction system in MATLAB fuzzy logic toolbox, shown in Figure 5 (a). The output of this scenario shows that it has winning chances of $71 \%$ as shown in Figure 5 (b).

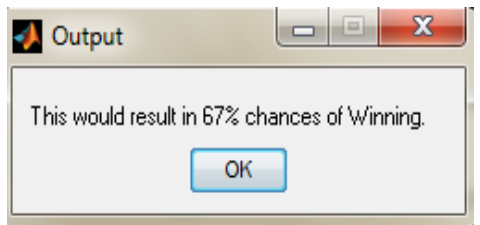

(b)

Fig. 4 (a): Parameters entered into Prediction Evaluator based on Scenario 1. (b) Output of Scenario 1. 


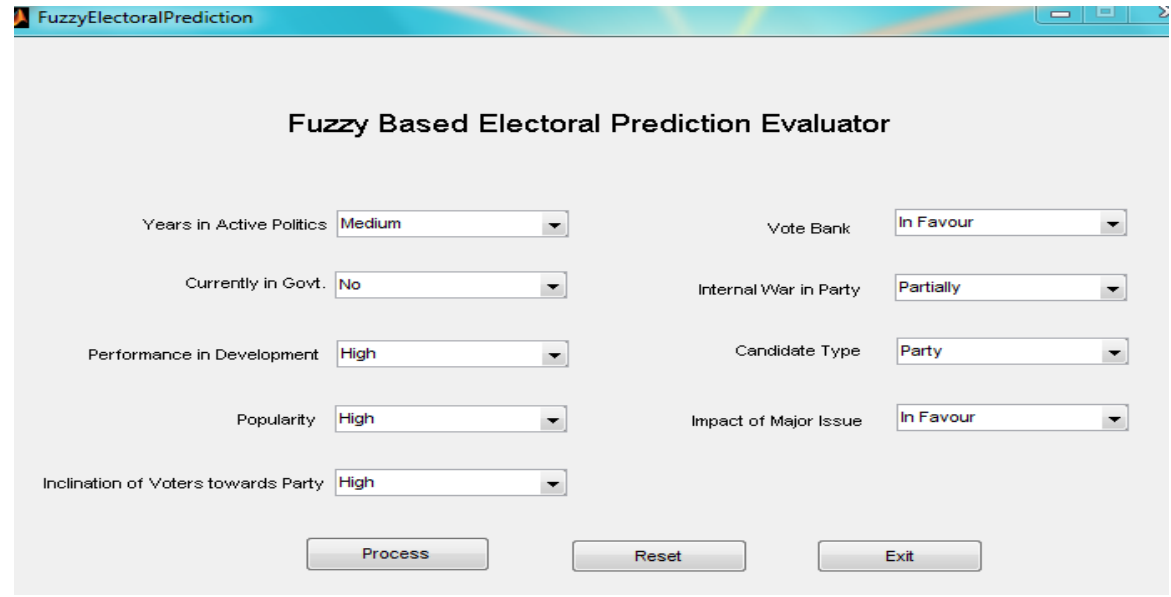

(a)

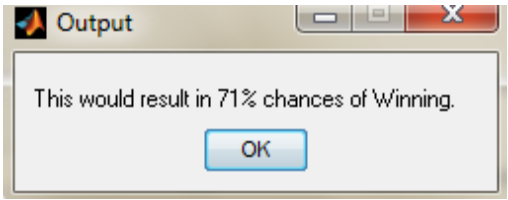

(b)

Fig. 5 (a): Parameters entered into Prediction Evaluator based on Scenario 2. (b) Output of Scenario 2.

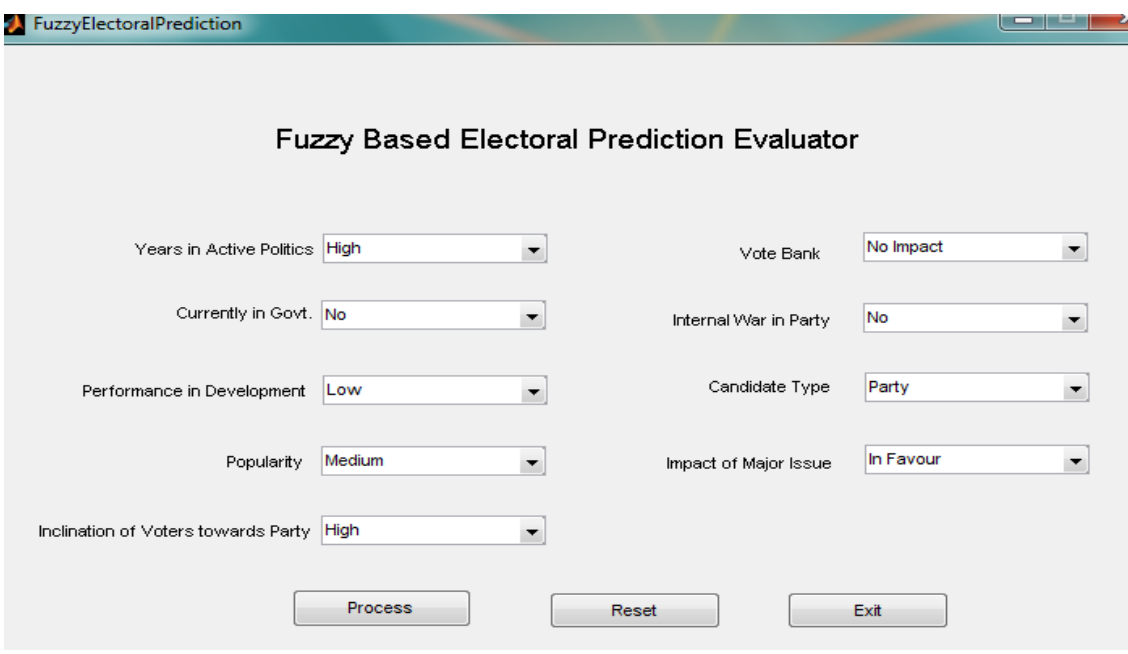

(a)

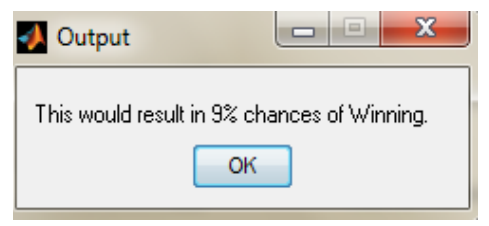

(b)

Fig. 6 (a): Parameters entered into Prediction Evaluator based on Scenario 3. (b) Output of Scenario 3.

Scenario 3: A candidate has high no of years in active politics and currently he does not belong to ruling party. i.e opposition party. He has very low performance in development as compared to scenario 2. Again the popularity is also medium and vote bank has no impact on the candidate it may be favor or opposite. He acts as a party candidate. There is no internal war in the party and all the major issues are in his favor. Due to the low performance of development, medium popularity and no exact position of voters chances of winning are very less as compared to previous cases. All these arguments are entered into the fuzzy logic based prediction system in MATLAB fuzzy logic toolbox, shown in Figure 6 (a). The output of this scenario shows that it has winning chances of $9 \%$ as shown in Figure 6 (b).
Scenario 4: A candidate has low no of years in active politics and currently he belongs to ruling party but he has low performance in development, rather popularity is high but vote bank is against for candidate and he acts as a party candidate. There is a partially internal war in the area and all the major issues are not in favor of candidate. Due to the low performance of development, major issue against and against value of vote bank the chances of loosing are very high. All these arguments are entered into the fuzzy logic based prediction system in MATLAB fuzzy logic toolbox, shown in Figure 7 (a). The output of this scenario shows that it has losing chances of $83 \%$ as shown in Figure 7 (b). 


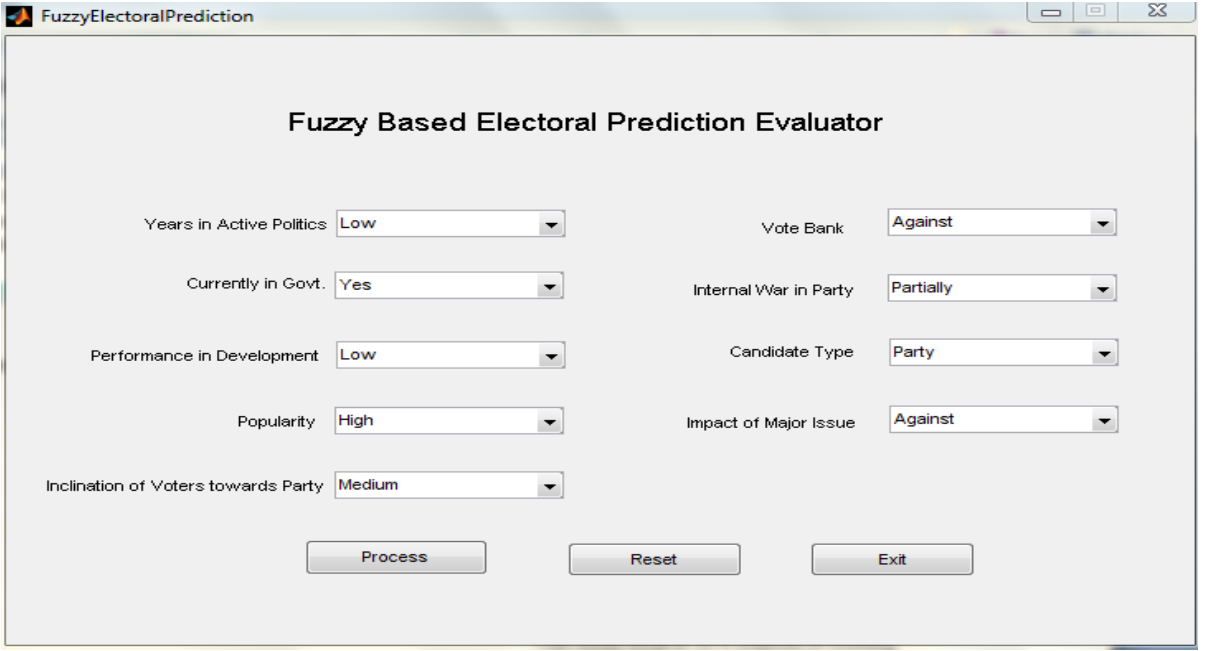

(a)

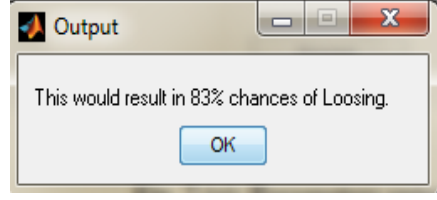

(b)

Fig. 7 (a): Parameters entered into Prediction Evaluator based on Scenario 4. (b) Output of Scenario 4.

Scenario 5: A candidate has low numbers of years in active politics and currently he also belongs to ruling party and also he has high performance in development as compared to scenario 4 , popularity and vote bank are also not in favor and he acts as an independent candidate. There is a partially internal war in the party and all the major issues have no impact. Due to the candidate type as independent the chances of losing are less than the scenario 4. All these arguments are entered into the fuzzy logic based prediction system in MATLAB fuzzy logic toolbox, shown in Figure 8 (a). The output of this scenario shows that it has loosing chances of $5 \%$ as shown in Figure 8 (b).

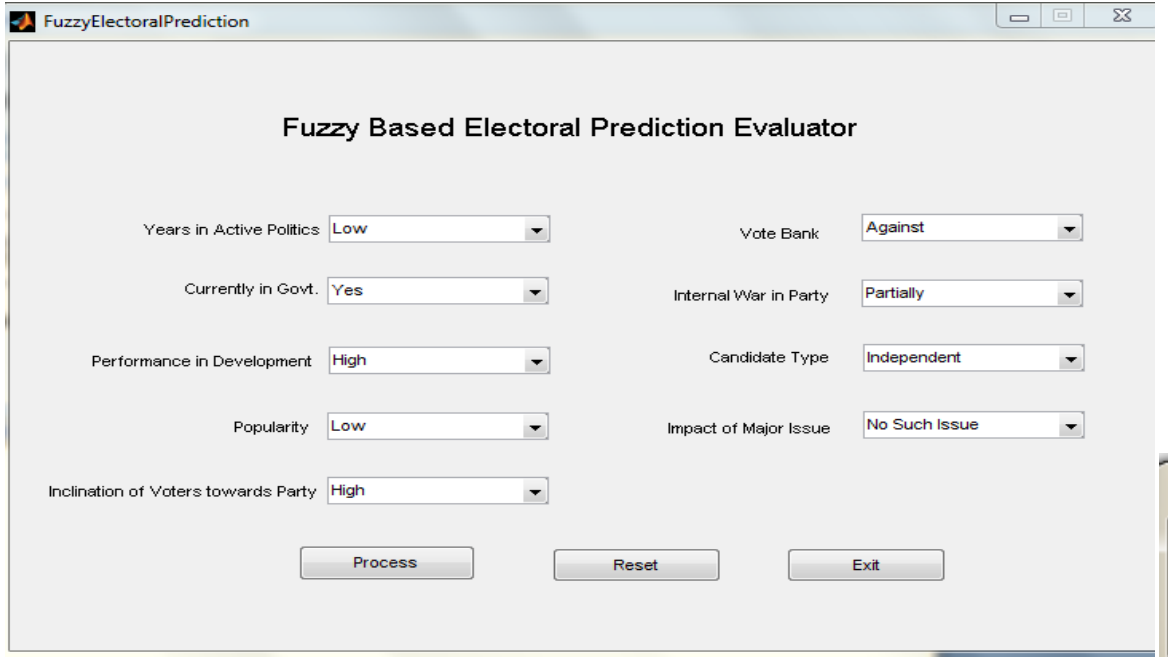

(a)

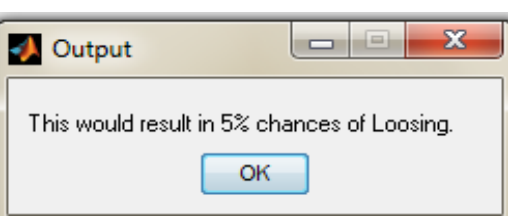

(b)

Fig. 8 (a): Parameters entered into Prediction Evaluator based on Scenario 5. (b) Output of Scenario 5.

\section{CONCLUSION}

This paper evaluates the prediction of a candidate in any type of election. To perform this task, a Fuzzy Inference System is used in MATLAB which takes into account all the arguments that must affect the prediction (winning chances as well as the losing chances) of a candidate. Each parameter is defined by membership functions. Then 91 different rules are made based on "if-then" conditions. Finally, three different scenarios of winning chances are hypothetically assumed and two different scenarios of losing chances are hypothetically assumed. This proposed model calculates the percentage of winning chances and also losing chances in the election.

This research can be used for future scope to calculate the more accurate prediction in the field of election as well as the areas other than the election. If number of inputs parameters can be increased or some other parameters can be added which actually effects the prediction then same model with little modification can give the accurate result for any kind of 
prediction. Prediction is an area of that type which never has its end. It means a regular and good research is always in demand which can be used to calculate the exact prediction in any area.

\section{REFERENCES}

[1] Zadeh, L. A. (1965). Fuzzy sets. Information and Control. 8, 338-353.

[2] Homaifar, A. and McCormick, E. (May 1995). Simultaneous design of membership functions and rule sets for fuzzy controllers using genetic algorithms. IEEE Trans. Fuzzy Systems. 3 (2).

[3] Mendel, J. M. (March 1995). Fuzzy logic systems for engineering: a tutorial. In Proceedings of the IEEE. Vol. 83. No. 3.

[4] Yao, Y. Y. (1996). Two views of the theory of rough sets infinite universes. International Journal of Approximation Reasoning. 15, 291-317.

[5] Yao, Y. Y. (1998). A comparative study of fuzzy sets and rough sets. Information Sciences. 109 (1-4), 227242.

[6] Hayward, G. and Davidson, V. (2003). Fuzzy logic applications. Analyst. 128, 1304-1306.

[7] Wang, W. and Bridges, S. M. (March 2000). Genetic algorithm optimization of membership functions for mining fuzzy association rules. In Proceedings of The International Joint Conference on Information Systems, Fuzzy Theory and Technology Conference.

[8] Abraham, A. (2005). Adaptation of fuzzy inference system using neural learning. Fuzzy System Engineering: Theory and Practice. N. Nedjah, Ed. et al. Berlin, Germany: Springer-Verlag, 3, 53-83.

[9] Shafiq, M. Z., Farooq, M. and Khayam, S. A. (2008). A comparative study of fuzzy inference systems. Neural Networks and Adaptive Neuro Fuzzy Inference Systems for Portscan Detection. EvoCOMNET, LNCS

[10] Zhiyi, F. (March 2004). A fuzzy inference system for synthetic evaluation of compost maturity and stability. Masters of Engineering thesis, University of Regina, Saskatchewan.

[11] Kumar, S., Bhatia, N. and Kapoor, N. (Feb 2011). Fuzzy logic based tool for loan risk prediction. In Proceedings of International Conference on Communication and Computing Technologies (ICCCT-2011), 180-183.

[12] Kumar, S., Bhatia, N. and Kapoor, N. (2011). Software risk analysis using fuzzy logic. International Journal of Computer Information Systems, 2 (2), 7-12.

[13] Singh Gursharan, Bhatia N. and Singh Sawtantar (2011). Fuzzy logic based cricket player performance evaluator. IJCA Special Issue on "Artificial Intelligence Techniques - Novel Approaches \& Practical Applications" AIT.

[14] M. J. Smithson, G. Oden.(1999). Fuzzy set theory and application in psychology, in D. Dubios \& H. Prade (Eds), International Handbook of Fuzzy Sets and Possibility Theory, Vol. 5, Kluwer, Amsterdam.

[15] J. Russell, M. Bullock, (1986). Fuzzy Concepts and the perception of emotion in facial expression, SocialCognition, Vol. 4, 309-341.
[16] V. Dimitrov,(1997). Use of Fuzzy Logic when dealing with Social Complexity, Complexity International, Vol. 04

[17] T. Yahashita, (1998). On a support system for human decision making by the combination of fuzzy reasoning and fuzzy structural modeling, Fuzzy Set and System, Vol. 8, 257-263

[18] Luis Teran, (2011). A Fuzzy based advisor for election and creation of political communities, Information System Research Group. Vol. 3,

[19] J.E Campbell, J.C. Garand (Eds.) (2000). Before the Vote: Forecasting American National Election, Sage Publication, Inc.

[20] J.E Campbell, (1992) Forecasting the presidential vote in the states, American Journal of Political Science 36 386407

[21] J.E Campbell, K.A. Wink, (1990) Trial-hear forecasts of the presidential vote, American Politics Quarterly 18 $251-269$

[22] M.S Lewis-Beck, T.W. Rice, (1984) forecasting presidential election: a comparison of native models, politics behavior $6,9-21$

[23] M.S Lewis-Beck, T.W. Rice, (1984). Forecasting U.S House Election, Legislative Studies Quarterly 9, 475-486

[24] M.S Lewis-Beck, T.W. Rice, (1992). Forecasting Elections, Congressional Quarterly Press, Washington DC.

[25] R. Fair, (1988). The effects of economic events on votes for president : Update, Political Behavior 10, 168-179

[26] G.F Royes, R.C Bastos, (2001). Fuzzy Sets in Political Science. In :IFSA World Congress and 20th NAFISPS International Conference, Vol. 2, 935-940

[27] Riley, J. (2005). Evolving fuzzy rules for goal-scoring behaviour in a robot soccer environment, PhD Thesis, RMIT University: Melbourne, Australia.

[28] Yanik, P., Ford, G. and McDaniel, W. (2010). An introduction and literature review of fuzzy logic applications for robot motion planning. In Proceedings of ASEE Southeast Section Conference.

\section{AUTHORS PROFILE}

Harmanjit Singh received his Graduate degree in Arts in 1998 and Master's degree in Information Technology in 2007 from Punjab Technical University, Jalandhar, India along with Master's degree in Computer Application in 2008 from Punjab Technical University, Jalandhar, India. He is working as Assistant Professor in Department of Computer Science, Punjab College of Technical Education (PCTE), Baddowal, Ludhiana. $\mathrm{He}$ is also pursuing Ph.D from Singhania University, Rajasthan, India. He has seven research papers to his credit. His areas of interest are fuzzy logic, graph theory and SEO. He is also associated with various international journals as reviewer

Dr Gurdev Singh received his Graduate degree in Arts in 2000 and Master's degree in Math in 2002 from Guru Nanak Dev University, Amritsar, India. He is working as Professor in Department of Information Technology, Gurukul Vidyapeeth 
Institute of Engineering \& Technology, Banur, Chandigarh. He has completed his Ph.D in 2010 in CSE. He has more than twenty research papers to his credit. His areas of interest are cloud computing, software metrics and fuzzy logic. He is also associated with various international journals as member of editorial team

Nitin Bhatia received his Graduate degree in Science in 1998 and Master's degree in Computer Application in 2001 from
Guru Nanak Dev University, Amritsar, India. He is working as Lecturer in Department of Computer Science, DAV College, Jalandhar. He is also pursuing Ph.D from Punjabi University, Patiala, India. He has twenty one research papers to his credit. His areas of interest are pattern recognition, computer vision and fuzzy logic. He is also associated with various international journals as reviewer 